expanded in the PB of pSS, display an in vivo activated Th17 phenotype, infiltrate MSG and are resistant to corticosteroids. Taken together, these data suggest a key role of this T-cell subset in the perpetuation of chronic sialoadenitis and eventually in SS prognosis and provide the clue to target DN T cells for therapeutic purposes in $\mathrm{pSS}$.

\section{A3.5 COMBINATION BLOCKING OF IL-6 AND IL-21 IN EXPERIMENTAL ARTHRITIS INHIBITS THEIR REDUNDANT ROLE IN TH17-DRIVEN JOINT PATHOLOGY}

doi:10.1136/annrheumdis-2013-203216.5

'Marije I Koenders, 'Debbie M Roeleveld, 1,2Renoud J Marijnissen, ${ }^{3}$ Cheryl NickersonNutter, 'Fons van de Loo, 'Wim B van den Berg. 'Radboud University Nijmegen Medical Centre, Rheumatology Research and Advanced Therapeutics, Nijmegen, the Netherlands; ' University Medical Centre Utrecht, the Netherlands; ${ }^{3}$ Pfizer Inc, Cambridge, Massachusetts, USA

Background and Objectives Both IL- 6 and IL- 21 have been described to drive in vitro Th17 differentiation in the presence of TGFbeta. We explored whether also in vivo IL- 6 and IL-21 play an exchangeable and redundant role in Th17 differentiation during experimental arthritis, and to what extent combined blocking of these cytokines inhibits Th17 differentiation and suppresses arthritis development.

Materials and Methods To investigate the synergistic effects of combined blocking of the IL-6 and IL-21 pathways, arthritis development and Th17 cells were first studied in IL-6 ${ }^{-} \times \mathrm{IL}^{-2} \mathrm{R}^{-1}$ mice and their wild-type (WT) and single-knockout controls during antigen-induced arthritis (AIA). In addition, the kinetic effects of combined IL-6R and IL-21 neutralisation was studied in DBA-1J mice treated at various stages of collagen-induced arthritis (CIA).

Results Mice deficient for either IL-6 or IL-21R showed suppressed antigen-induced arthritis compared to WT controls. This disease reduction was accompanied by a significant reduction in CD4+IL17+ $\mathrm{T}$ cells in the draining lymph nodes as determined by FACS However, mice lacking both the IL- 6 and IL-21 signalling pathways showed an even stronger disease suppression than the IL- $6 \%$ and IL-21 $\mathrm{R}^{-}$mice, and a striking reduction in Th17 levels was observed in these mice.

Based upon our findings in gene-knockout mice, we aimed to confirm the synergistic effects of IL-6/IL-21 with a cytokineneutralisation approach using anti-IL-6R antibodies and sIL-21R-Fc treatment during CIA. Antibodies were given as single treatment or in combination, and was started at immunisation (day 0 ) or around the booster (day 21).

Combined blocking of IL-6R and IL-21 early during arthritis development (day 0) was a very potent approach to prevent arthritis development, reaching a disease incidence of only $40 \%$ at day 35 (isotype control 100\%, sIL-21R-Fc 100\%, anti-IL-6R 60\%). Analyzing the mice that did develop arthritis, we observed that the anti-IL-6R/ sIL-21R-Fc combination was also clearly more potent in suppressing the arthritis severity in comparison to the single treatments. Interestingly, blocking the IL-6/IL-21 pathways at a later stage during arthritis development (day 21) was clearly less effective and did not show any additional effects to anti-IL-6R treatment alone.

Conclusions Combined blocking of the IL- 6 and IL-21 pathways suppresses Th17 differentiation in vivo as demonstrated by our IL-6/IL-21R-deficient mice. However, our neutralisation study during CIA shows that to influence arthritis development this IL-6/ IL-21 blocking approach only has a limited therapeutic window. These findings suggests that to target Th17-driven joint pathology, blocking Th17 effector cytokines like IL-17 and IL-22 might be more effective than attempting to reduce Th17 cell numbers during active disease.

\section{A3.6 COMPARATIVE ANALYSIS OF THE THERAPEUTIC POTENTIAL OF INDUCIBLE TREG CELL POPULATIONS IN EXPERIMENTAL MODEL OF ARTHRITIS}

doi:10.1136/annrheumdis-2013-203216.6

'Julie Quentin, 'Delphine Martire, 'Anne-Laure Mausset-Bonnefont, ${ }^{2}$ Hélène Asnagli, ${ }^{2}$ Nathalie Belmonte, ${ }^{2}$ Arnaud Foussat, 'Christian Jorgensen, 'Rita Vincente, 'Pascale Louis-Plence. 'INSERM U844, Université Montpellier 1, CHU Lapeyronnie, Montpellier, France; ${ }^{2} T x C e l l$, Allée de la Nertière, Les Cardoulines, 06560 Valbonne-Sophia Antipolis, France

Background and Objectives Adoptive cell transfer of Treg cells is a promising approach to restore tolerance in autoimmune disease. However the various type of Tregs, their doses of injection and their in vivo-suppressive mechanism need to be precisely define to clearly establish which Tregs will be able to dampen efficiently the immune response in the various settings.

In our study, we compared the therapeutic potential of IL10secreting Tregs: $\mathrm{Tr} 1$ and CD49b-induced Tregs and $\mathrm{CD}_{2} 5^{+} \mathrm{FoxP} \mathrm{P}^{+}$ Tregs. These two Treg populations share several phenotypic markers as well as immunosuppressive properties. In the present study we perform adoptive cell transfer experiments of the various inducible Treg cells in order to compare their impact on the immune response. Materials and Methods CD49b Treg cells were generated in naive mice following repetitive injections of immature dendritic cells (DC). Cell sorting experiments were realised to obtain $98 \%$ pure CD49b T cells and CD25+ cells. Collagen type II (bCII) specific Tr1 clones were obtained from TCR transgenic mice and expanded in vitro. Selected clones showed in vitro antigen specificity, $\operatorname{Tr} 1$ cytokine profile and IL10- and TGF $\beta$-dependent suppressive activity. Several doses of CD49b or $\operatorname{Tr} 1$ cells were injected i.v. at day 28 in established collagen-induced arthritis. Clinical signs of arthritis were scored, as well as the in vivo impact on the proliferation of Teffector cells and the maturation of DC.

Results We defined for both Treg cell populations the most efficient dose in curative settings experiments. One single dose of $3 \times 10^{6}$ or $1 \times 10^{6}$ of $\operatorname{Tr} 1$ cell administration could reduce the incidence and severity of CIA. Interestingly, higher dose of $10 \mathrm{M}$ of $\operatorname{Tr} 1$ cells did not improve the disease. In the same manner, the dose of $10^{5}$ CD4CD49b ${ }^{+}$cells reverse clinical symptom with a lack of efficacy of higher doses. We demonstrated following injection of Treg cells a clear impact on the proliferation of the effector cells in vivo.

Conclusions Our results suggest that even if the Treg cells present some similarities, we need to precisely define the dose and type of Treg that will be efficient in each experimental setting. We provide also a comparative analysis of the in vivo mechanism responsible of the protection of the various subtypes of Treg cells.

\section{A3.7 COMPARISON OF THE EFFECTS OF TH17 AND TH1 CELLS ON ENDOTHELIAL CELLS AND SYNOVIOCYTES}

doi:10.1136/annrheumdis-2013-203216.7

'Arnaud Hot, 'Laura Maggi, 'Fabien Lavocat, ${ }^{2}$ Francesco Annunziato, 'Pierre Miossec ${ }^{1}$ Department of Clinical Immunology and Rheumatology; Immunogenomic and Inflammation Research Unit EA 4130, Hopital Edouard Herriot, University of Lyon, France; ${ }^{2}$ Department of Internal Medicine and DENOTHE Centre, University of Florence, Firenze, Italy

Background Interaction between different types of T-cells and surrounding non-hematopoietic cells is essential for the proper function of the immune system. Here, we focus on the synoviocytes from RA patients and Endothelial Cells (EC) and compare their interaction with Th1 and Th17 cells.

Methods To assess the interaction of T cells with stromal cells, the effects of Th1 or Th17 cytokines, Th1 or Th17 clone supernatants and coculture of Th clones and stromal cells were analysed. HUVEC (Human Umbilical Vein Endothelial Cells) were used as a model for EC and synoviocytes were isolated from synovium from RA 
patients. $\mathrm{CD}^{+} \mathrm{T}$ cells, derived from PBMCs of healthy donors, were used to obtain $\mathrm{T}$-cell clones. $\mathrm{CD}^{+}{ }^{+} \mathrm{T}$-cell clones were classified on the basis of their ability to produce IFN- $\gamma$ and/or IL-17. T cell clones were polyclonally stimulated with anti-CD3/CD28. Cytokine expression was assessed with RT-PCR and production in supernatants by ELISA.

Results As previously described, IL-17A and TNF- $\alpha$ had a synergistic effect for both cells. Moreover, a stronger induction for IL-6 production was seen for synoviocytes compared to EC $(1005 \pm 22 \mathrm{pg} / \mathrm{ml}$ versus $250 \pm 15 \mathrm{ng} / \mathrm{ml}$ respectively). Supernatants from inactivated Th1 or Th17 clones had no effect on EC. Regarding synoviocytes, supernatants from inactivated clones induced IL- 6 and IL- 8 mRNA with a stronger effect for Th17 cells (1000 fold versus 100 fold compared to resting synoviocytes, $p=0.045)$. While activated Th1 supernatants had a strong effect on EC and increased the expression of IL-6, IL-8, E-selectin, and tissue factor mRNA (98.12, 89 fold respectively, compare to control), Th17 supernatants had no effect. Activated Th1 and Th17 supernatants had the same effect on synoviocytes (254 and 754 fold compared to control). Th1 cells were more potent inducers of IL-6, IL-8 and tissue factor mRNA in EC than Th17 which had no significant effect $(5,12.5$, 7.5 fold respectively compared to control). Th17 cells were most effective to stimulate IL- 6 and IL-8 mRNA expression in synoviocytes compared to Th1 (100 fold versus 24 for IL-6 mRNA, $p=0.027$ ). Anti-IL-17 antibody reduced IL-6 production from $20.0 \pm 4.5 \mathrm{ng} / \mathrm{mL}$ to $13.2 \pm 3.9$ $\mathrm{ng} / \mathrm{ml}(\mathrm{p}=0.032)$ in synoviocytes cultured with Th17 clones.

Conclusions While Th1 cells were able to induce inflammatory cytokine expression in EC and synoviocytes, Th17 cells were only active to promote inflammation in inflammatory cells such as synoviocytes. The effect of Th17 cells appears to depend of IL-17 and the type of stromal cells.

\section{A3.8 EXTRATHYMIC AUTOIMMUNE REGULATOR (AIRE) EXPRESSION IN RHEUMATOID ARTHRITIS}

doi:10.1136/annrheumdis-2013-203216.8

AR Noort, KPM van Zoest, MC Lebre, PP Tak, SW Tas. Division of Clinical Immunology \& Rheumatology, Academic Medical Center, University of Amsterdam, The Netherlands

Background AIRE is a transcription factor that is involved in the negative selection of self-reactive thymocytes in the thymus. Recently, AIRE protein has also been detected in peripheral lymphoid organs, predominantly in dendritic cells (DC). In these peripheral sites, AIRE was found to regulate the expression of a group of tissue-specific antigens that is distinct from those expressed in the thymus, suggesting that peripheral AIRE may play a complementary role in tolerance induction. It is currently unknown whether AIRE may play a role in inflamed tissues, such as rheumatoid arthritis (RA) synovial tissue (ST).

Objective To document and further characterise extrathymic AIRE expressing cells in ST and paired peripheral blood (PB) mononuclear cells (MCs) as well synovial fluid (SF) MCs of RA patients. Methods ST was obtained via mini-arthroscopy from inflamed joints of RA or undifferentiated arthritis (UA) patients. Expression of AIRE was evaluated using IHC and IF microscopy. AIRE expression was also investigated in PB and SF DC using flow cytometry. Results AIRE expressing cells were detected in $80 \%$ of analysed RA ST and in contrast only in $25 \%$ of UA ST. Further characterisation using double-immunofluorescence microscopy revealed that these cells were predominantly CD1c+ myeloid (m)DC. Interestingly, a significantly higher percentage of $\mathrm{CD} 1 \mathrm{c}+\mathrm{mDC}$ in RA SF expressed AIRE $(55 \pm 5 \%)$ compared to RA PB $(20 \pm 3 \%)$ and healthy PB $(19.7 \pm 2 \%)$

Conclusions Extrathymic AIRE expressing $\mathrm{CD} 1 \mathrm{c}+\mathrm{mDC}$ are present in RA ST and RA SF, suggesting a role in synovial inflammation. Extrathymic AIRE expression in RA synovial inflammation may be an attempt to control inflammation through the induction of peripheral tolerance to antigens involved in the perpetuation of the chronic inflammatory response.

\section{A3.9 IL-17RA SIGNALLING IS ESSENTIAL FOR COLLAGEN INDUCED ARTHRITIS DEVELOPMENT}

doi:10.1136/annrheumdis-2013-203216.9

1.20dilia BJ Corneth, 1,2Adriana MC Mus, 1,2Patrick S Asmawidjaja, 1,2Maarten D Brem, ${ }^{1,2}$ Franka Luk, ${ }^{1,2}$ Wendy Dankers, ${ }^{3}$ Rudi W Hendriks, ${ }^{1,2}$ Erik Lubberts. ${ }^{1}$ Departments of Rheumatology; ${ }^{2}$ mmunology; ${ }^{3}$ Pulmonary Medicine, Erasmus MC, University Medical Center, Rotterdam, The Netherlands

Introduction IL-17A plays an important role in collagen-induced arthritis (CIA). It signals through the IL-17 receptor (IL-17R) A and $\mathrm{C}$ heterodimer. The IL-17RA appears to be a common receptor subunit for several IL-17 cytokine family members, including IL-17A, IL-17C and IL-17F. Lack of IL-17RA signalling may therefore have a broader effect than lack of IL-17A alone. We therefore aim to determine the role of IL-17RA signalling in arthritis.

Methods Disease incidence and severity were scored in wild type (positive control), IL-17RA deficient and IL-23p19 deficient (negative control) mice in CIA. T helper cell profiles and humoral immune responses were analysed by flow cytometry and immuno-histochemistry. Serum auto-reactive IgG antibodies were measured by ELISA. Pathogenicity of $\mathrm{T}$ cells and total splenocytes was determined in a functional assay in vitro.

Results As expected, wild type mice developed CIA from day 21 after the first immunisation. IL-23p19 deficient mice did not develop arthritis. Interestingly, IL-17RA deficient mice were completely protected against CIA, even after a third immunisation. This is in contrast to IL-17A deficient mice, of which $20 \%$ is still susceptible to CIA. T cells in IL-17RA deficient mice display a Th2-like phenotype in CIA with higher proportions of IL-4 producing CD4 T cells. This population is distinct from IL-17A producing $\mathrm{T}$ cells. The shift in T cell phenotype induces a less inflammatory B cell response with fewer plasma cells in the spleen and lower pathogenic IgG2a antibody production in favour of IgG1 production. In a functional assay, both isolated CD4+ T cells and total splenocytes of IL-17RA deficient mice were less capable of inducing pro-inflammatory IL-6 production by normal, IL-17RA expressing synovial fibroblasts in CIA in vitro.

Conclusions Here we show that lack of IL-17RA signalling prevents auto-immune inflammation of the joint. In addition, $T$ helper cells shift to a Th2 like phenotype characterised by IL-4 production. $\mathrm{T}$ cells and splenocytes of these mice are less pathogenic, leading to lower pathogenic IgG2a antibody levels in serum. This is in contrast with IL-17A deficient mice and suggests that other factors involved in CD4 + T cell differentiation and pathogenicity can signal through the IL-17RA.

\section{A3.10 IL-6 RECEPTOR BLOCKADE ENHANCES CD39+ REGULATORY T-CELL DEVELOPMENT IN RHEUMATOID ARTHRITIS AND IN EXPERIMENTAL ARTHRITIS}

doi:10.1136/annrheumdis-2013-203216.10

'Allan Thiolat, 'Jerome Biton, 'Luca Semerano, ${ }^{2} Y$ ves-Marie Pers, ${ }^{2}$ Pierre Portales, ${ }^{1}$ Delphine Lemeiter, ${ }^{1}$ Patrice Decker, ${ }^{2}$ Christian Jorgensen, 'Marie-Christophe Boissier, 2Pascale Louis-Plence, 'Natacha Bessis. 'EA4222, Li2P, University Paris 13, Sorbonne Paris Cité and Rheumatology Department, Avicenne Hospital, Assistance PubliqueHôpitaux de Paris (AP-HP), Bobigny, 93009 France, Bobigny, France; 2INSERM U844, CHU Saint-Eloi, Université Montpellier 1, CHU Lapeyronie, Montpellier, France

Background and Objectives Studies have demonstrated the clinical efficacy of tocilizumab, a humanised anti-IL-6 receptor (R) antibody $(A b)$, in patients with rheumatoid arthritis (RA). The rational 